\title{
Spinning Molecules, Spinning Spins: Modulation of an Electron Spin Exchange Interaction in a Highly Anisotropic Hyperfine Field
}

\author{
Alexander M. Brugh, Ruobing Wang, Michael J. Therien,* and Malcolm D. E. Forbes*
}

Cite This: ACS Omega 2021, 6, 27865-27873

Read Online

ABSTRACT: An investigation of spin and conformational dynamics in a series of symmetric $\mathrm{Cu}-\mathrm{Cu}$ porphyrin dimer solutions is presented using electron paramagnetic resonance (EPR) spectroscopy. Previous spectral simulations focused on the isotropic exchange interaction $\left(J_{\text {avg }}\right)$ between the $\mathrm{Cu}$ centers. In this work, an additional line broadening parameter $\left(J_{\text {mod }}\right)$ is explored in detail via variable temperature X-band EPR in liquid solution for several different structures. The $J_{\text {mod }}$ phenomenon is due to fluctuations in the spin exchange interaction caused by conformational motion of the porphyrin planes. The $J_{\text {mod }}$ parameter scales with the inverse of the rotational barriers that determine the Boltzmann-weighted torsional angle distribution between neighboring porphyrin planes. Arrhenius plots allow for extraction of the activation energies for rotation, which are 5.77,
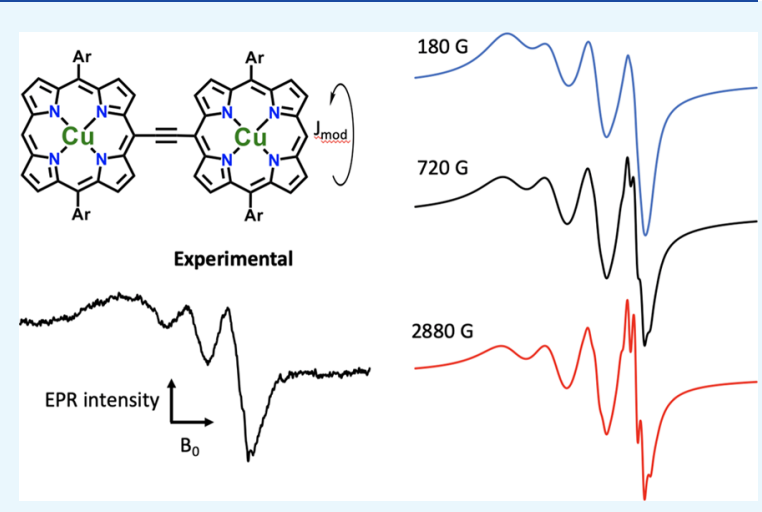
2.84 , and $5.31 \mathrm{~kJ} / \mathrm{mol}$ for ethyne-bridged (porphinato)copper(II)(porphinato)copper(II), butadiyne-bridged (porphinato)copper(II)(porphinato)copper(II), and ethyne-bridged (porphinato)copper(II)-(porphinato)zinc(II)-(porphinato)copper(II) complexes, respectively. DFT calculations of these torsional barriers match well with the experimental results. This is the first report of a $J_{\text {mod }}$ analysis within a highly anisotropic hyperfine field and demonstrates the utility of the theory for extraction of dynamic information.

\section{INTRODUCTION}

Biradicals and related open shell structures have long fascinated chemists and physicists due to their complex stereoelectronic properties and the insight they provide into electron correlation and predictions of molecular structure. ${ }^{1}$ Indeed, theorists and computational chemists remain challenged to develop methods that provide accurate structures, overall energies, and spin state information simultaneously. ${ }^{2,3}$ Investigation of biradical and biradicaloid structures and spin properties are receiving renewed interest in the fields of spintronics and quantum information science (QIS), ${ }^{4}$ where they may be of significant utility in the successful transmission of quantum spin information over long distances.

Conjugated porphyrin arrays with multiple transition metals have been extensively investigated both theoretically and experimentally due to their extraordinary optoelectronic properties and tunable molecular structures. ${ }^{5,6}$ Their facile synthesis allows for the incorporation of several different transition-metal ions into a molecular framework, while maintaining a well-defined geometry. When electron spin exchange interactions are considered, there are fewer examples in the literature for such arrays, which represent relatively isolated two-spin systems. Magnetic susceptibility measurements typically assess bulk properties rather than individual isotropic exchange interactions. This sparse literature on the topic of rigid biradicals or biradicaloids is particularly true for systems involving two metal centers coupled to a rigid spacer.

Ethyne-bridged multi-porphyrin arrays, ${ }^{7-21}$ such as the dimer structures shown in Figure 1, are ideal for systematic studies of electron spin exchange interactions in systems containing rigid conjugated spacers. The molecules depicted in Figure 1 can be synthesized with different spacer lengths, attachment points, and a variety of central metal ions. Electron spin exchange couplings have been reported previously in similar structures to give information about magnetism, spin delocalization, metal-ligand $\mathrm{d}-\pi$ mixing, and spin transmission pathways. ${ }^{22,23}$ We have recently presented two studies of such structures where we explored the metal-metal spinspin coupling in porphyrin array frameworks that make possible highly delocalized electronic structures. ${ }^{20,24}$

In many ways, "spin chemistry" phenomena such as chemically induced dynamic electron spin polarization $(\mathrm{CIDEP})^{25}$ and magnetic field effects, ${ }^{26,27}$ which were

Received: July 3, 2021

Accepted: September 22, 2021

Published: October 18, 2021 


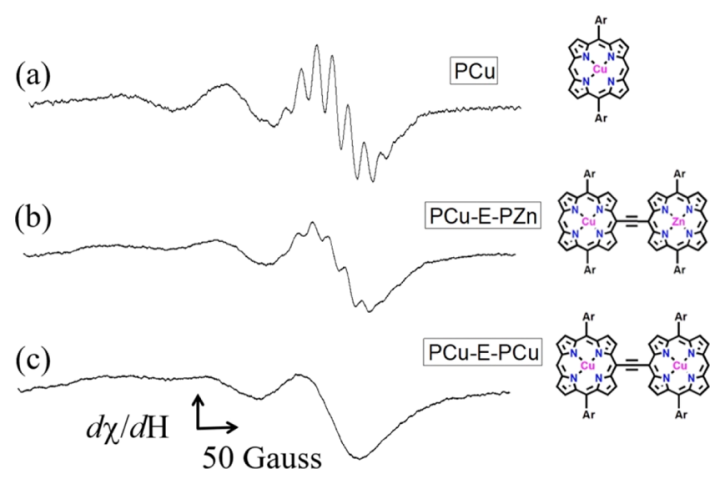

Figure 1. Examples of $\mathrm{Cu}(\mathrm{Zn})$ - porphyrin monomers and dimers and their unusual EPR spectra, obtained in toluene solution at a $\sim 3 \mu \mathrm{M}$ concentration. (a) Typical $\mathrm{Cu}$ porphyrin monomer, (b) porphyrin dimer with one $\mathrm{Cu}$ center, and (c) porphyrin dimer with two $\mathrm{Cu}$ centers.

extensively investigated in the latter half of the 20th century, kindled the fire for the strong present interest in the simultaneous movement of spin and charge and subsequent storage of relevant spin information. The alkyne-bridged (porphinato)copper(II) complex shown at the bottom of Figure 1 is analogous to an organic biradical, of which many have been studied extensively using both steady-state and timeresolved electron paramagnetic resonance (EPR). ${ }^{28}$ However, there are three major differences between these previous studies of purely organic structures and those involving these systems: (1) the large $\mathrm{Cu}$ nuclear hyperfine interaction; (2) the high anisotropy of this interaction; and (3) the lack of many torsional degrees of freedom, such as those found in polymethylene chain biradicals and biradicaloids.

The structures and spectra in Figure 1 also help to define the logic of the current paper and to stress what is new. The top structure is essentially a monoradical $\mathrm{Cu}$-porphyrin, of which many have been studied before and for which simulation routines have long been available. The most prominent feature of the spectrum is the broadening of the hyperfine lines from $\mathrm{Cu}$ : in particular, the low-field transitions are significantly broader than the high-field lines (the multiplet structure at a high field is due to the ${ }^{14} \mathrm{~N}$ atoms coordinated to copper). Our simulation routine, described in more detail below, easily accounts for the monoradical spectrum. The middle structure in the figure shows a similar spectrum to that at the top, but the extra (non-paramagnetic) porphyrin ring adds additional homogeneous line width by slowing down the tumbling rate. Again, this is easily accounted for by spectral simulation. The bottom structure and spectrum show the dramatic effect of adding a second paramagnetic porphyrin ring. Comparison of the middle and bottom spectra show that for dimers of a similar size, significant additional line broadening from electron spin exchange is present. The fact that all three spectra can be precisely simulated ${ }^{20,24}$ using the monomer as a platform for the hyperfine-induced broadening, molecular volume for homogeneous broadening, and spin exchange for additional broadening through interactions with another spin demonstrates the "buildup" of the simulation routine for more complex relaxation phenomena, to which we now turn.

The X-band liquid solution EPR spectra of $\mathrm{Cu}-\mathrm{Cu}$ porphyrin dimers of the type shown in Figure 1 are affected by the molecular dynamics mentioned above, that is, the torsional/librational motion of the porphyrin planes about the linear alkyne bridge. They can also tumble along their long axis. In either case, their motional dynamics in solution are simpler than those of related bis[(porphinato)metal] systems bridged by alkyl or other flexible chains due to their restricted degrees of conformational freedom (e.g., their end-to-end distance is fixed). The two types of motion are depicted in Figure 2 for the ethynyl-bridged dimer. In the end-over-end tumbling motion shown in Figure 2A, no major changes to the value of the isotropic exchange interaction are expected. This motion might, however, play a role in the modulation of the electronic dipole-dipole interaction either directly as an additional splitting in the spectrum or as a line shape

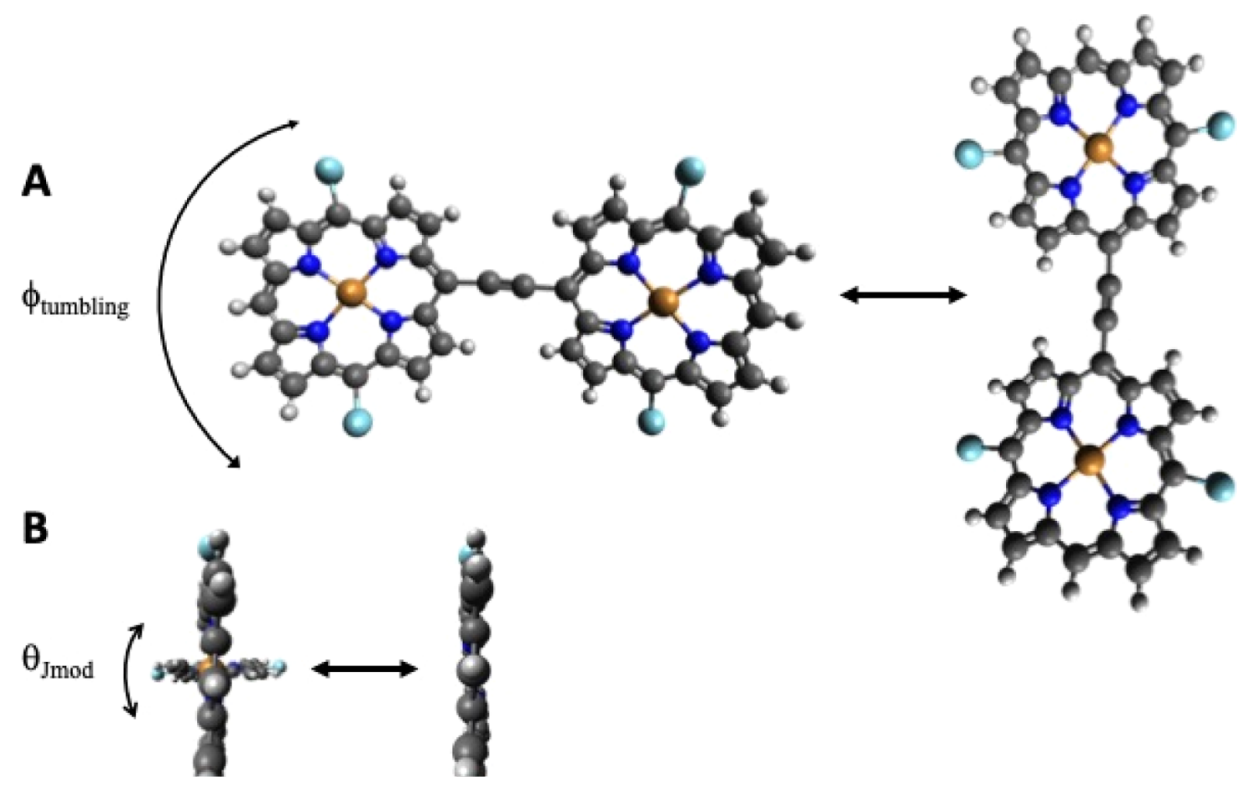

Figure 2. Two modes of molecular motion for Cu-porphyrin dimers in solution. (A) End-over-end tumbling (relatively slow). (B) Paddle wheel (torsional) angle changes between the rings containing the unpaired electrons (relatively fast). The motion in (B) is expected to play a role in determining both the absolute value and the dynamics of the exchange interaction. 
parameter (vide infra). ${ }^{29}$ However, the "paddle-wheel" type motion illustrated in Figure 2B, which modulates the overlap integral between the radical centers, should have a large impact on the measured electronic spin-spin couplings and is expected to be faster due to a lower rotational barrier, consistent with the Stokes-Einstein-Debye relation. ${ }^{30}$ The dynamic processes depicted in Figure 2 should have quite different time scales. The end-over-end tumbling motion requires the displacement of many more solvent molecules than the paddle wheel motion, and as a result, it is expected to be at least an order of magnitude slower.

In each of our two recent papers on symmetric copper porphyrin dimers linked by ethynyl, butadiynyl, and oligoynyl spacers, simulation of their steady-state EPR (SSEPR) spectra allowed us to extract the isotropic spin exchange parameter $\left(J_{\text {avg }}\right)$, which significantly broadens the dimer spectra. ${ }^{20,24}$ Additionally, a dynamic relaxation term $\left(J_{\text {mod }}\right)$ was included in the simulation model, which accounts for additional broadening due to the temperature-dependent modulation of the Boltzmann-weighted torsional angle distribution between the porphyrin rings. The theory section of Supporting Information derives and gives detailed definitions of $J_{\text {avg }}$ and $J_{\text {mod. }}$. For all alkyne-bridged (porphinato)copper(II) arrays studied to date, with increasing temperature, $J_{\text {avg }}$ decreases, while $J_{\text {mod }}$ increases. These effects can be explained by the temperaturedependent conformational distributions evident in these systems. As the temperature increases, the Boltzmannweighted average of conformations, differentiated by the porphyrin-porphyrin interplanar torsional angles, increases for all alkyne-bridged bis $[($ porphinato $) \mathrm{Cu}]$ arrays. As this population-weighted average porphyrin-porphyrin interplanar torsional angle increases, the corresponding porphyrinporphyrin electronic coupling decreases. The topic of electronic delocalization will be revisited in a later section.

Dependable simulations of these spectra are further complicated by the presence of a very large anisotropy in the $\mathrm{Cu}$ hyperfine tensor, which affects the line shape at a lower field dramatically. ${ }^{31,32}$ The use of ethyne and butadiyne spacers provides a high sensitivity between the spacer and the metal in terms of orbital interactions. This has been demonstrated in our previous papers ${ }^{20,24}$ as the basis for a strong temperature dependence of $J_{\text {avg }}$ in such complexes. In this paper, we present the finer details of our simulation procedures and show how $J_{\text {mod }}$ contributes to the overall line shape. We also place our line shape calculation routine in context with examples of $J$ modulation in other systems. To the best of our knowledge, this is the first analysis of the $J$ modulation phenomenon in a symmetric system but one that contains a highly anisotropic hyperfine field, making it a unique case in dynamic EPR spectroscopy.

\section{THEORY}

To understand the effects of $J$ modulation in the highly anisotropic hyperfine field of the copper-porphyrin dimer system in Figure 1, some basic equations are necessary. SSEPR spectra are accomplished by (1) selecting an appropriate spin Hamiltonian and basis set, (2) diagonalizing the Hamiltonian to obtain its eigenvalues, and (3) calculating the resonant EPR frequencies and transition probabilities from the energy differences for the allowed transitions using the standard selection rule: an allowed transition has a change in electron spin quantum number of \pm 1 and a change in nuclear spin quantum number of $0 .{ }^{33}$ Additional details of the theory are presented in Supporting Information and in additional refs 34-3536373839. The theory leads to the derivation of two very simple expressions (eqs 1 and 2) for the relevant electron spin relaxation times, of which is eq 2 is the most relevant for line shape analysis

$$
\begin{aligned}
& T_{1}^{-1}=4\left\langle V^{2}\right\rangle \tau_{\mathrm{e}}\left(q^{2} / \Omega^{2}\right) \\
& T_{2}^{-1}=2\left\langle V^{2}\right\rangle \tau_{\mathrm{e}}\left(1 \pm J_{\mathrm{avg}} / \Omega\right)
\end{aligned}
$$

where

$$
\begin{aligned}
& \Omega=\left\{J_{\text {avg }}^{2}+q^{2}\right\}^{1 / 2} \\
& q=\left[\beta B_{0}\left(g_{1}-g_{2}\right)+\sum\left(a_{i} m_{i}-a_{j} m_{j}\right)\right] / 2
\end{aligned}
$$

As noted in Supporting Information, the spin Hamiltonian used is from the Dirac formalism for spin exchange and leads to an expression for the singlet-triplet energy gap of $-2 J_{\text {avg }}$, with a singlet ground state when the value of $J_{\text {avg }}$ is negative. The term $q$ in eq 4 represents the off-diagonal element of the spin Hamiltonian responsible for mixing the singlet and middle triplet levels of the biradicaloid. The term $V^{2} \tau_{\mathrm{e}}$ in eq 2 defines the line width addition due to modulation of the exchange interaction, that is, $J_{\text {mod }}$. It is expressed in Gauss to make its units compatible as an addition to the "natural" line width. The $V$ term represents the maximum possible fluctuation of the $\mathrm{J}$ value at any instant, and $V^{2}$ times the torsional rotational correlation time $\tau_{\mathrm{e}}$ gives the maximum contribution to the line width of any particular transition caused by this relaxation mechanism. Note that when the mixing term $q$ goes to 0 , the effect of $J_{\text {mod }}$ on $T_{1}$ vanishes. For $T_{2}$, important for the $J_{\text {mod }}$ process, the situation is more complex. A value of $q=0$ in eq 4 leads to either the term vanishing $(1-J / \Omega$ option $)$ or a maximum contribution to the line width from $J_{\text {mod }}$ (the $1+J$ / $\Omega$ option). The former applies to transitions involving states with predominantly singlet character, and the latter applies to transitions involving states with predominantly triplet character (see Supporting Information for further details).

The $J$ modulation phenomenon dates back to some of the earliest days of EPR spectroscopy, particularly the pioneering work of Rassat and Rosantzev, ${ }^{40,41}$ who synthesized and characterized some of the first nitroxide radicals and biradicals. Indeed, these early papers, especially on biradicals, have enjoyed additional recent interest from the spintronics and quantum information science communities; similarly, nitroxide radicals and biradicals find great utility as dynamic nuclear spin polarization agents in NMR spectroscopy. A comprehensive review by Hudson and Luckhurst ${ }^{42}$ brought the spectral features of $J$ modulation in nitroxides to a more general audience, summarized by the stickplot spectrum shown in Figure 3.

It is important to note that the energy level diagram in Figure 3 is specific for the case of $J_{\text {avg }}>q$, which means the transitions to and from predominantly singlet character states. It is also specific for a symmetric biradical structure with equal hyperfine coupling constants and $g$-factors on both radical sites. The spectrum is predicted from the tree diagram to present, at large $J_{\text {avg }}$ a quintet with half the monoradical hyperfine and a $1: 2: 3: 2: 1$ intensity ratio. The origin of the alternating line width phenomenon so highly characteristic of $J$ modulation effects emerges succinctly from this diagram. Nuclear spin sub-ensembles with symmetric orientations have $q=0$ and therefore no $T_{2}$ contribution from $J_{\text {mod }}$ relaxation. 


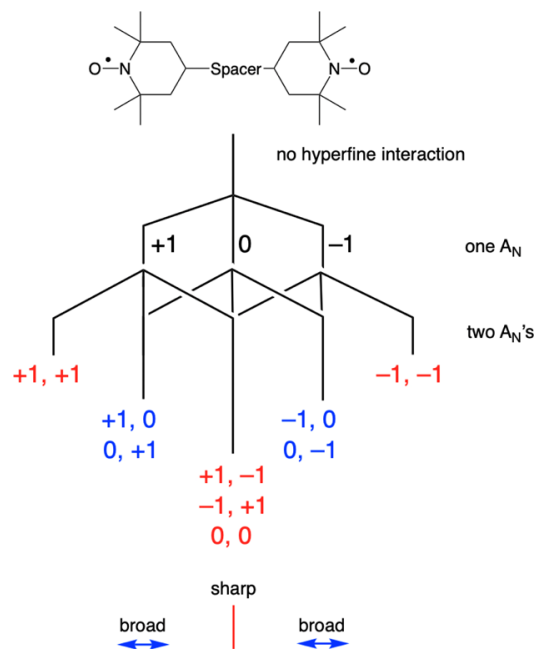

Figure 3. Tree diagram and line broadening due to $J_{\bmod }$ for a symmetric nitroxide biradical. Note that only transitions involving state $|2\rangle$ are observed, due to the large $J_{\text {avg }}$ value, and only states between which there is nuclear spin alignment (total nuclear spin quantum number, $M_{I}=0$ and $M_{I}= \pm 1$ ) are broadened, due to symmetry considerations.

For metal complexes, asymmetric line broadening even in the absence of $\mathrm{J}$ modulation can be significant. To account for the "monoradical" anisotropy present in $\mathrm{Cu}$ (II) system EPR spectra, an empirical equation devised by Wilson and Kivelson was also adapted to our simulation program. ${ }^{31,32}$ The line width $\left(\Delta B^{\mathrm{D}}\right)$ is a function of the $z$-component of the nuclear spin of copper $\left(m_{\mathrm{I}}\right)$. The various constants $\left(k_{0-2}\right)$ represent the extent of interactions within the nuclear and electronic and $g$ tensors. These constants are fitting parameters that were determined through spectral simulation of a monoradical species, a copper(II) porphyrin similar to the top structure in Figure 1. The ratio of monomer and dimer rotational correlation times is represented by $\tau_{\mathrm{C}}^{\mathrm{D}} / \tau_{\mathrm{C}}^{\mathrm{M}}$. This ratio was determined by Toyama, Asano-Someda, and Kaizu and adjusted experimentally for our samples ${ }^{43}$

$$
\Delta B^{\mathrm{D}}=\frac{\tau_{\mathrm{c}}^{\mathrm{D}}}{\tau_{\mathrm{c}}^{\mathrm{M}}}\left(k_{0}+k_{1} m_{I}+k_{2} m_{I}^{2}\right)
$$

The SSEPR spectrum of a $\mathrm{Cu}(\mathrm{II})$ porphyrin is a quartet of nonets due to interactions with the $\mathrm{Cu}$ nuclear spin $(I=3 / 2)$ and the four $\mathrm{N}$ atoms $(I=1)$ surrounding it. The asymmetric hyperfine line broadening affects the low-field line shape significantly more than the high-field lines.

An important question that arises is whether broadening due to hyperfine anisotropy and broadening due to $J_{\text {mod }}$ can be distinguished. In these systems, we have a fortunate case where the two contributions can indeed be separated due to the symmetry of the spin system. The hyperfine anisotropy term defined in eq 5 affects each hyperfine line in the spectrum differently due to its dependence on $m_{I}$. The overall effect of eq 5 is that low-field lines are broadened significantly more than high-field lines. This is a characteristic of EPR spectroscopy for $\mathrm{Cu}$ and other metal complexes and is exemplified by the top and middle spectra in Figure 1 . On the other hand, the $J_{\text {mod }}$ process leads to alternating line widths across the spectrum as explained by Figure 3 and the surrounding text. Since these terms are applied independently (as they must be) in the simulation routine, we can identify broadening from each process with successful simulations. Furthermore, there is strong evidence that the anisotropy-induced relaxation described by eq 5 shows only a small dependence on temperature $\left(1-5 \%\right.$ changes in line width over $\left.80{ }^{\circ} \mathrm{C}\right)$, whereas the $J_{\text {mod }}$ process shows a much stronger temperature dependence. A closer look at how exactly $J_{\text {mod }}$ influences the line shape is therefore of interest as it opens the gate for a more quantitative assessment of at least some of the molecular dynamics described in Figure 2. It should be noted that the $J_{\text {mod }}$ term itself can be further parsed into the fluctuating perturbation matrix element $V$ and its correlation time. This

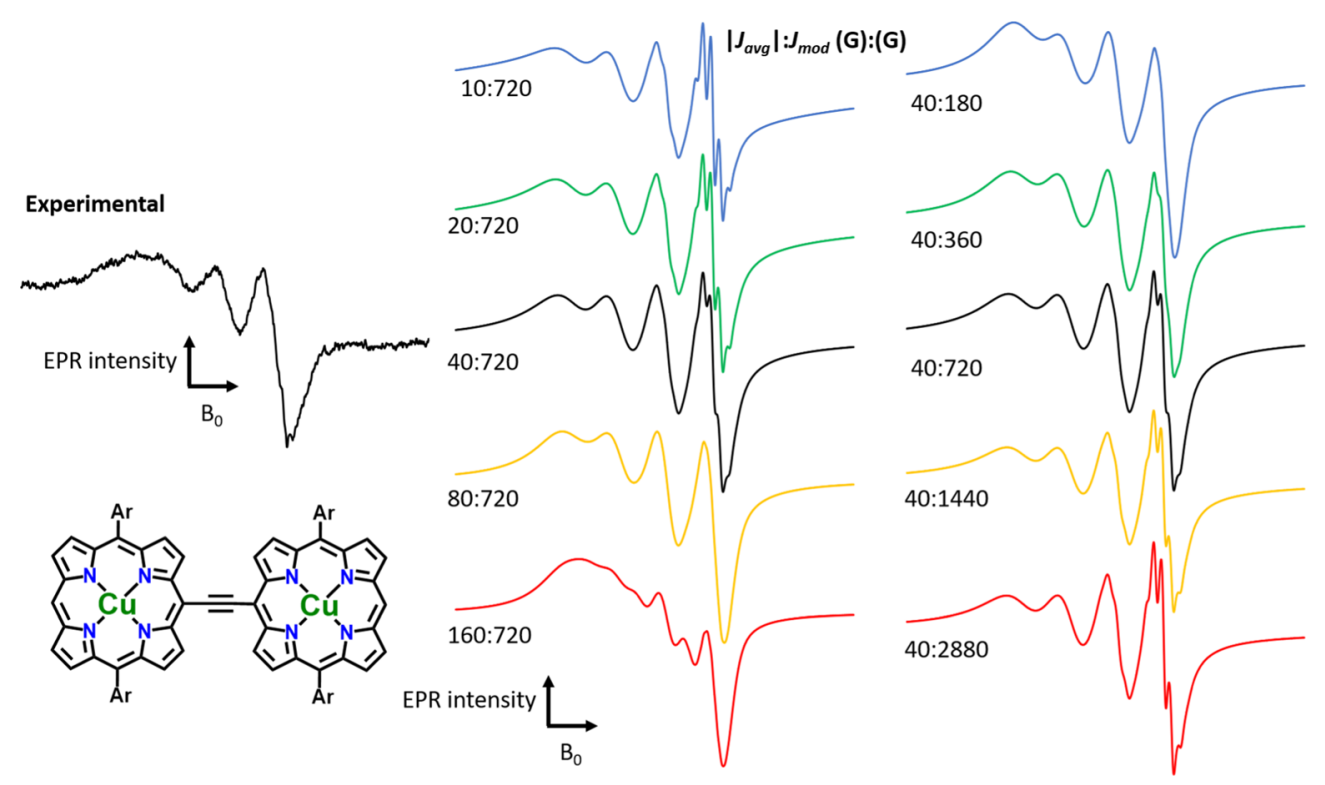

Figure 4. Experimental and simulated spectra for $\mathrm{PCu}-\mathrm{E}-\mathrm{PCu}$ in toluene at $343 \mathrm{~K}$. In the center, simulations are presented with increasing values of $J_{\text {avg }}$ with a fixed $J_{\text {mod }}$, and on the right, simulations are presented with increasing values of $J_{\text {mod }}$ and a fixed $J_{\text {avg }}$. The middle spectrum in both columns is the best fit simulation with $J_{\text {avg }}$ of $40 \mathrm{G}$ and $J_{\bmod }$ of $720 \mathrm{G}$. Note especially the sensitivity of the high-field lines to both parameters but at opposite extremes. 
allows us to continue building the story as it opens the opportunity for analysis of temperature-dependent data and therefore activation barriers.

\section{RESULTS AND DISCUSSION}

Here, we present an analysis of simulations of several representative systems in the context of $J$ modulation theory. An immediate concern is that while the complexes show significant structural symmetry, the anisotropy of the $\mathrm{Cu}$ hyperfine tensors disrupts this symmetry considerably. The strong dependence of the Kivelson line shape parameters ${ }^{31,32}$ on the $\mathrm{Cu}$ nucleus quantum number $(-3 / 2,-1 /,+1 / 2$, and $+3 / 2$ ) always renders the low-field lines in the spectrum much broader than those at a high field. This means that alternating line widths may be difficult to observe, and the requirement for a $J_{\text {mod }}$ parameter in the simulation may be more subtle than in more isotropic systems.

In Figure 4, we compare the effect of increasing $J_{\text {mod }}$ or $J_{\text {avg }}$ while holding the other parameter constant from the best fit (middle spectrum in Figure 4). The different biradicaloids were selected to show the variation of the spectral features as a function of end-to-end distance and temperature. A particularly noteworthy feature in all three figures is that the high-field multiplets from the nitrogen hyperfine couplings become more well-resolved as $J_{\text {avg }}$ decreases but also as $J_{\text {mod }}$ increases. These dependences clearly demonstrate that the best fit requires a balance of these parameters, one reflecting the static picture and the other accounting for the molecular dynamics. Section S2 of Supporting Information contains additional examples at different temperatures.

Another interesting feature is that even the best fits fail somewhat for the lower field transitions, and this is worthy of some discussion. There are several reasons why our simulation program might fail: (1) it should be understood that the Wilson-Kivelson monoradical line shape parameters $\left(k_{0}, k_{1}\right.$, and $k_{2}$ in eq 5) are an approximation based on perturbation theory and may break down for some systems. (2) The Redfield limit for the $J_{\text {mod }}$ theory is technically only valid for the fast motion limit, although we believe that we are in this limit for most cases. (3) Our program ignores the electron spin dipole-dipole interaction, which may be present to some extent, perhaps not as an observable splitting in the spectrum but as a broadening of selected transitions via spin relaxation. The tumbling motion depicted in Figure 2A could be very effective in adding a fluctuating dipolar coupling to the spin relaxation manifold, and this is likely to affect spin angular momentum vectors already in a highly anisotropic environment. The fact that the fits at a low field become slightly better when the temperature is increased for the same biradicaloid supports the latter hypothesis.

It is instructive to consider electron density in more detail, particularly its role in determining the magnitude of $J_{\text {avg }}$. Less delocalization gives rise to a smaller average exchange interaction. Since the singlet-triplet energy gap is proportional to the measured exchange interaction $\left(\Delta E_{\mathrm{ST}}=-2 J_{\mathrm{avg}}\right)$, the impact of the extent of delocalization on the magnitude of the exchange interaction can be modeled using calculated values of the singlet-triplet energy gaps at different conformations.

Density functional theory sometimes has difficulty providing high-quality results for the energies of open shell molecules, especially biradicals and specifically singlet-state biradicals. This is a topic of significant interest in the computational chemistry community. ${ }^{44}$ Older semi-empirical methods such as
MNDO, CNDO, or PM6, while more accurate in determining energies of organic biradicals in various spin states, cannot be used successfully in these systems because the metal centers are poorly parameterized. From Hückel theory, it is known that the singlet-triplet energy gap in a rigid biradical with only one degree of rotational freedom will vary with $\cos ^{2} \theta$, where $\theta$ is the dihedral angle between the two p orbitals containing the unpaired electrons.

For the complexes studied here, we can make a qualitative estimate of the variation in the value of $\Delta E_{\mathrm{ST}}=-2 J$ if we have a starting point value for $\Delta \mathrm{E}_{\mathrm{ST}}$ at a dihedral angle between the porphyrin planes of $0^{\circ}$, where the energy gap will be maximized, and simply multiply this value by $\cos ^{2} \theta$ to give conformational dependence at any other dihedral angle. Our " $0{ }^{\circ}$ results" for $\mathrm{PCu}-\mathrm{E}-\mathrm{PCu}$ and $\mathrm{PCu}-\mathrm{EE}-\mathrm{PCu}$ using unrestricted DFT methods provide values for $\Delta E_{\mathrm{ST}}$ of 940 and 94 Gauss, respectively, with the singlet state lower in energy than the triplet for both complexes. The use of DFT for these "starting point" calculations, while not ideal, will at least allow us to establish a model for the experimentally observed trends for the variation of $J$ with temperature.

The results of our calculations of the magnitude of $\Delta E_{\mathrm{ST}}$ for $\mathrm{PCu}-\mathrm{E}-\mathrm{PCu}$ and $\mathrm{PCu}-\mathrm{EE}-\mathrm{PCu}$ at each dihedral angle of every $10^{\circ}$ from 10 to $90^{\circ}$, as described above, are shown in Figure 5.

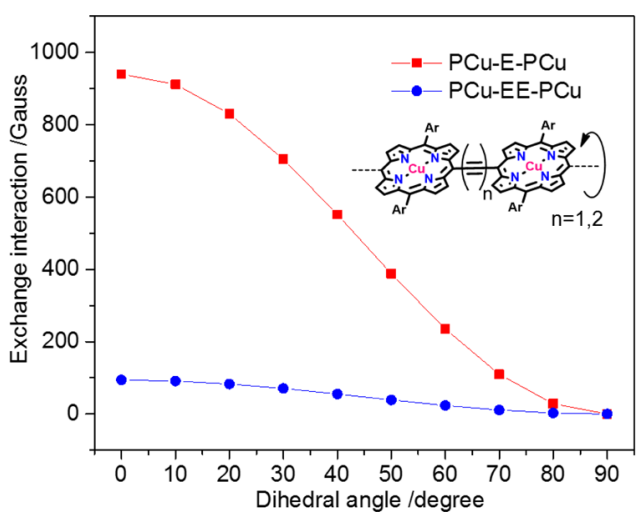

Figure 5. Calculated singlet-triplet energy gap $\left(\left|\Delta E_{\mathrm{ST}}\right|\right)$ for $\mathrm{PCu}-\mathrm{E}-$ $\mathrm{PCu}($ red, $n=1$ ) and $\mathrm{PCu}-\mathrm{EE}-\mathrm{PCu}$ (blue, $n=2$ ) at different dihedral angles from 0 to $90^{\circ}$.

The experimentally observed exchange interaction is an average over all conformers present in the solution, and the distribution of conformers should obey a Boltzmann distribution. Thus, we can simulate the experimental observed temperature dependence of the average exchange interaction using eq 6

$$
J_{\text {avg }}(T)=\sum_{i=1}^{10}\left(p_{i}(T)^{*} J_{i}\right)
$$

Here, $p_{i}$ is the probability of a particular conformation having a $J$ value of $J_{i}$, calculated using the Boltzmann distribution and the magnitude of the exchange interaction of the $i_{\text {th }}$ conformer at temperature $T$. The Boltzmann factors are calculated from the DFT results, and the summation to 10 covers the 10 data points for the dihedral angles represented in Figure 5. The experimental and corresponding simulated results for $J_{\text {avg }}$ are shown in Figure 6. Clearly, the simulated results are consistent with the experimental trend that $J_{\text {avg }}$ decreases with increasing 

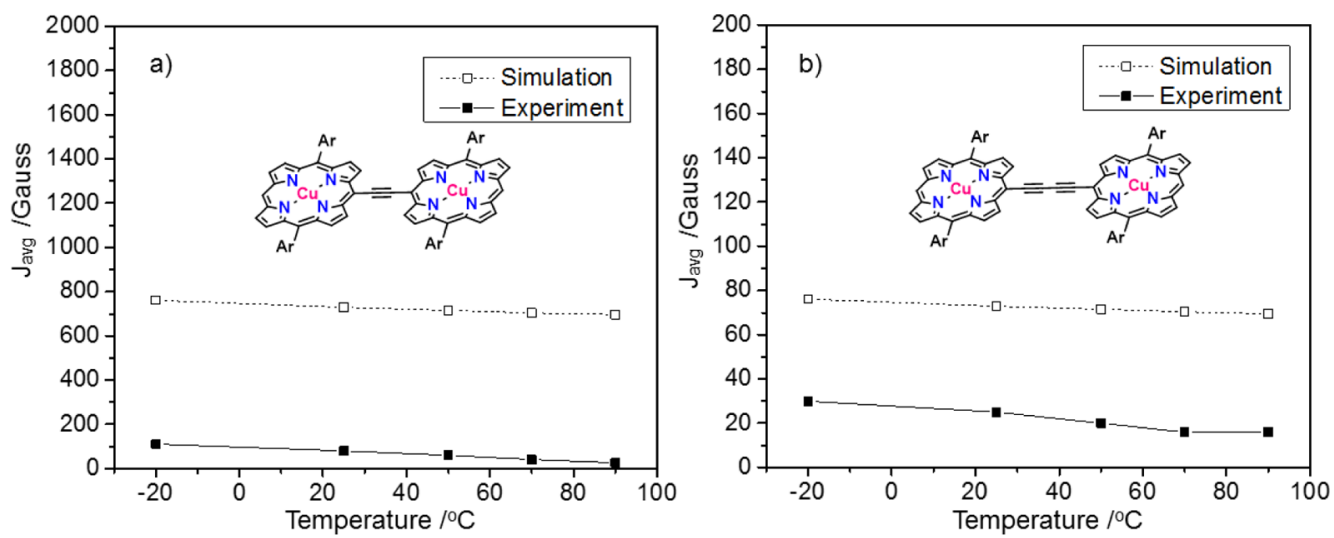

Figure 6. Simulated and experimental $J_{\text {avg }}$ of (a) $\mathrm{PCu}-\mathrm{E}-\mathrm{PCu}$ and (b) $\mathrm{PCu}-\mathrm{EE}-\mathrm{PCu}$ at different temperatures. Note the drastically different vertical scales in the two plots.

temperature for both the $\mathrm{PCu}-\mathrm{E}-\mathrm{PCu}$ and $\mathrm{PCu}-\mathrm{EE}-\mathrm{PCu}$ structures. Especially noteworthy is that the unrestricted DFT calculations for the "starting point" $0^{\circ}$ values fall in a reasonable range for $\mathrm{PCu}-\mathrm{E}-\mathrm{PCu}$ and $\mathrm{PCu}-\mathrm{EE}-\mathrm{PCu}$, and the factor of 10 difference in $J$ between the two structures is also adequately reproduced.

Another interesting feature from simulation parameters is that at each temperature, the value for $J_{\text {mod }}$ in $\mathrm{PCu}-\mathrm{EE}-\mathrm{PCu}$ is higher than that for either $\mathrm{PCu}-\mathrm{E}-\mathrm{PCu}$ or $\mathrm{PCu}-\mathrm{E}-\mathrm{PZn}-$ $\mathrm{E}-\mathrm{PCu}$, but the overall range of $J_{\text {mod }}$ values is narrower. ${ }^{20} \mathrm{On}$ the other hand, the range of $J_{\text {avg }}$ values for $\mathrm{PCu}-\mathrm{EE}-\mathrm{PCu}$ is smaller than those for $\mathrm{PCu}-\mathrm{E}-\mathrm{PCu}$ and $\mathrm{PCu}-\mathrm{E}-\mathrm{PZn}-\mathrm{E}-$ $\mathrm{PCu}$. These differences derive from the fact that the barrier to rotation about the butadiyne bridge is diminished with respect to that for the ethyne bridges of $\mathrm{PCu}-\mathrm{E}-\mathrm{PCu}$ and $\mathrm{PCu}-\mathrm{E}-$ $\mathrm{PZn}-\mathrm{E}-\mathrm{PCu}{ }^{20,24,45-47}$ Thus, the conformational distribution for the butadiyne-bridged molecules will change less with temperature. As a result, both $J_{\text {avg }}$ and $J_{\text {mod }}$ for the PCu-EE$\mathrm{PCu}$ structure are less dependent on temperature than those for the other dimer structures.

This difference in behavior between the butadiyne- and the ethyne-bridged dimers can be modeled by generating porphyrin-porphyrin dihedral-angle-dependent energy maps of $\mathrm{PCu}-\mathrm{EE}-\mathrm{PCu}$ and $\mathrm{PCu}-\mathrm{E}-\mathrm{PCu}$ dimers (Figure 7). It can be seen that the calculated rotational barrier for $\mathrm{PCu}-\mathrm{EE}-$ $\mathrm{PCu}(2.69 \mathrm{~kJ} / \mathrm{mol})$ is $1.87 \mathrm{~kJ} / \mathrm{mol}$ lower than that calculated for $\mathrm{PCu}-\mathrm{E}-\mathrm{PCu}(4.56 \mathrm{~kJ} / \mathrm{mol})$. Since the magnitude of $J_{\bmod }$ scales with the correlation time for porphyrin plane rotation, an Arrhenius plot (Figure 8) will give a slope representing the activation energy for this rotation (i.e., the rotational barrier).

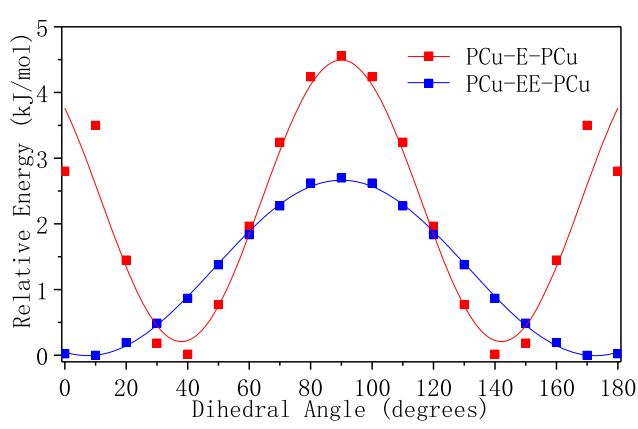

Figure 7. Porphyrin-porphyrin dihedral-angle-dependent energy maps of $\mathrm{PCu}-\mathrm{EE}-\mathrm{PCu}$ and $\mathrm{PCu}-\mathrm{E}-\mathrm{PCu}$ dimers obtained by DFT calculations at the 6-31G level and fitted by a sine function.

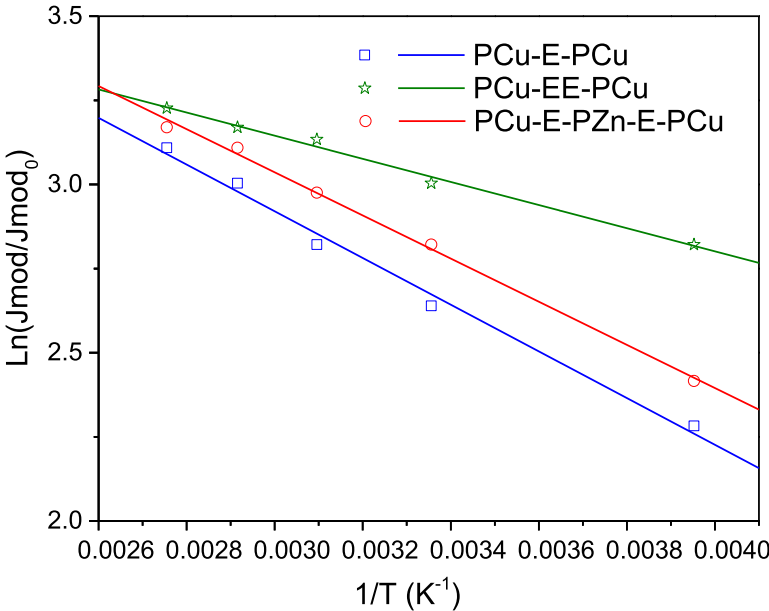

Figure 8. Arrhenius plots $\left(\ln \left(J_{\bmod } / J_{\bmod 0}\right)\right.$ vs $\left.1 / T\right)$ of $\mathrm{PCu}-\mathrm{E}-\mathrm{PCu}$ (blue), $\mathrm{PCu}-\mathrm{EE}-\mathrm{PCu}$ (green), and $\mathrm{PCu}-\mathrm{E}-\mathrm{PZn}-\mathrm{E}-\mathrm{PCu}$ (red).

Analysis of the Arrhenius plots in Figure 6 gives activation energies for porphyrin plane rotation in $\mathrm{PCu}-\mathrm{E}-\mathrm{PCu}, \mathrm{PCu}-$ $\mathrm{EE}-\mathrm{PCu}$, and $\mathrm{PCu}-\mathrm{E}-\mathrm{PZn}-\mathrm{E}-\mathrm{PCu}$ of $5.77,2.84$, and 5.31 $\mathrm{kJ} / \mathrm{mol}$, respectively. This is consistent with the results of these DFT calculations for $\mathrm{PCu}-\mathrm{E}-\mathrm{PCu}$ and $\mathrm{PCu}-\mathrm{EE}-\mathrm{PCu}$. It is observed that $\mathrm{PCu}-\mathrm{E}-\mathrm{PCu}$ and $\mathrm{PCu}-\mathrm{E}-\mathrm{PZn}-\mathrm{E}-\mathrm{PCu}$ have similar slopes (i.e., activation energies for plane rotation), which is reasonable as both these complexes feature a meso-tomeso ethyne bridging linkage topology between the porphyrin planes. The rotational barriers of alkyne-bridged (porphinato)copper(II) complexes, which are extracted from the simulation for SSEPR spectra of these complexes, are consistent with previous theoretical ${ }^{45-48}$ and experimental ${ }^{49}$ investigations regarding the rotation barrier of alkyne-bridged (porphinato)zinc(II) analogues.

The activation barriers can be used in the Arrhenius equation to extract rates for the paddle wheel motion. The inverse values of these rates give the correlation time for the torsional motion process. Using a typical pre-exponential factor of $10^{10} \mathrm{~s}^{-1}$, the obtained correlation times are $1.0,0.3$, and 0.8 ns, respectively (see Supporting Information for further details). Furthermore, we can use these correlation times to estimate the value of the fluctuation in the exchange interaction $V$ in our fitting parameter $V^{2} \tau_{\mathrm{e}}$. The value of $V$ is thus estimated to be approximately $500 \mathrm{G}$ for $\mathrm{PCu}-\mathrm{E}-\mathrm{PCu}$ and $\mathrm{PCu}-\mathrm{E}-\mathrm{PZn}-\mathrm{E}-\mathrm{PCu}$, meaning that the maximum 
fluctuation in $J$ is 5-10 times $J_{\text {avg }}$. This is a very reasonable number for the perturbation of $J$ via torsional motion when one considers variations in the dihedral angle $\left(\cos ^{2} \theta\right.$ varies from about 1 to 0.1 for $\theta$ varying from 0 to $70^{\circ}$, almost the complete possible range of values).

Although it is tempting to plot the measured $J_{\text {avg }}$ values versus distance and look for temperature variations in the falloff parameter for spin exchange, it should be noted that both $J_{\text {avg }}$ and $J_{\text {mod }}$ are functions of distance and temperature and hence may not necessarily be monotonic functions with similar distance trends. Indeed, such plots fail to show much correlation for precisely this reason: too many parameters affecting the EPR spectral line shape change at once. Certainly, the delicate interplay of $J_{\bmod }$ and $J_{\text {avg }}$ parameter magnitudes required to fit the experimental data (Figures 4, S2, S3) is strong evidence that this is the case.

Our results clearly show the utility of rigid spacers for the evaluation of spin exchange couplings in biradicaloids. We note here especially that these structures are electronically neutral and both their dynamic and static properties can be probed without light excitation. This provides a rich landscape of information linking the dynamics of a biradicaloid to its spectroscopic features in a logical and quantitative fashion. Purely organic analogues of such structures would be more difficult to produce by any other methods than photochemically, and they would not be thermally stable. Our ability to extract accurate rotational barriers from an Arrhenius analysis shows an additional advantage of studying such structures in detail with high-resolution spectroscopy. The difference in the magnitude of $J_{\text {avg }}$ between $\mathrm{PCu}-\mathrm{E}-\mathrm{PCu}$ and $\mathrm{PCu}-\mathrm{EE}-\mathrm{PCu}$ at any given temperature further underscores the through-bond nature of the coupling in these systems and the larger electronic coupling provided by ethyne relative to butadiyne in these systems. $14,20,24,45$

In one of our systems $(\mathrm{PCu}-\mathrm{E}-\mathrm{PCu}), J_{\text {avg }}$ is of almost exactly the same magnitude as the $\mathrm{Cu}$ nuclear hyperfine interaction. A possible consequence of a situation where $J_{\text {avg }} \approx$ $A_{\mathrm{Cu}}$ is that the singlet-triplet mixing will be maximized (fully superimposed). This is the essence of an optimized qubit in quantum computing (QC). We suggest that the ease of synthesis of these systems provides a high degree of control for $\mathrm{read} /$ write processes in QC and other quantum information systems (QISs) that require molecular meta-architectures. It is hoped that this will inspire the next-generation design of useful structures for novel QISs.

\section{CONCLUSIONS}

$\mathrm{PCu}-\mathrm{E}-\mathrm{PCu}, \mathrm{PCu}-\mathrm{E}-\mathrm{PZn}-\mathrm{E}-\mathrm{PCu}$, and their related structures $^{20,24}$ can clearly be of great utility for the study of spin and magnetic effects in molecules and other hierarchal molecular materials; in such systems, conjugation motif, spacer size, and temperature are all important factors, with the latter two being the focus of this paper. The structures are symmetric, relatively easy to synthesize, and their EPR spectra can be recorded in a short time period with good signal-tonoise ratios over a wide temperature range. We can also successfully account for the expected order of magnitude difference in $J_{\text {avg }}$ between the two complexes with different spacers. Calculations support the experimental findings that $J_{\text {avg }}$ falls off exponentially with distance, but perfect correlations are difficult to find because several parameters affecting the EPR spectral line shape are involved. The temperature dependence of $J_{\text {avg }}$ can be successfully modeled with an understanding of the conformational energy landscape of the complexes and using the simple $\cos ^{2} \theta$ relationship that accounts for the variation of the singlet-triplet energy gap with dihedral angle.

\section{EXPERIMENTAL SECTION}

Characterization data and details regarding the syntheses of the $\mathrm{Cu}$-porphyrin dimers used for this study have been reported previously, as well as complete descriptions of the EPR spectral data collection. ${ }^{20,24}$

\section{ASSOCIATED CONTENT}

\section{(s) Supporting Information}

The Supporting Information is available free of charge at https://pubs.acs.org/doi/10.1021/acsomega.1c03490.

Theory of $J$ modulation and additional EPR spectral simulations (PDF)

\section{AUTHOR INFORMATION}

\section{Corresponding Authors}

Michael J. Therien - Department of Chemistry, French Family Science Center, Duke University, Durham, North Carolina 27708, United States; (1) orcid.org/0000-00034876-0036; Email: michael.therien@duke.edu

Malcolm D. E. Forbes - Center for Photochemical Sciences, Department of Chemistry, Bowling Green State University, Bowling Green, Ohio 43403, United States; (1) orcid.org/ 0000-0003-4040-386X; Email: forbesm@bgsu.edu

\section{Authors}

Alexander M. Brugh - Center for Photochemical Sciences, Department of Chemistry, Bowling Green State University, Bowling Green, Ohio 43403, United States

Ruobing Wang - Department of Chemistry, French Family Science Center, Duke University, Durham, North Carolina 27708, United States; 10 orcid.org/0000-0002-2414-8777

Complete contact information is available at:

https://pubs.acs.org/10.1021/acsomega.1c03490

\section{Notes}

The authors declare no competing financial interest.

\section{ACKNOWLEDGMENTS}

A.M.B. and M.D.E.F. would like to thank the National Science Foundation for support through grants CHE-1464817 and CHE-1900541. R.W. and M.J.T. would like to thank the Division of Chemical Sciences, Geosciences, and Biosciences, Office of Basic Energy Sciences, of the U.S. Department of Energy (DE-SC0001517) for research support. R.W. is grateful to Duke University for a Graduate Program Nanoscience Fellowship.

\section{REFERENCES}

(1) Borden, W. T. Diradicals; Wiley, 1982.

(2) Abe, M. Diradicals. Chem. Rev. 2013, 113, 7011-7088.

(3) Michl, J. Spin-Orbit Coupling in Biradicals. 1. The 2-Electronsin-2-Orbitals Model Revisited. J. Am. Chem. Soc. 1996, 118, 35683579 .

(4) Wasielewski, M. R.; Forbes, M. D. E.; Frank, N. L.; Kowalski, K.; Scholes, G. D.; Yuen-Zhou, J.; Baldo, M. A.; Freedman, D. E.; Goldsmith, R. H.; Goodson, T.; Kirk, M. L.; McCusker, J. K.; Ogilvie, J. P.; Shultz, D. A.; Stoll, S.; Whaley, K. B. Exploiting Chemistry and Molecular Systems for Quantum Information Science. Nat. Rev. Chem. 2020, 4, 490-504. 
(5) Castellano, M.; Ruiz-García, R.; Cano, J.; Ferrando-Soria, J.; Pardo, E.; Fortea-Pérez, F. R.; Stiriba, S.-E.; Julve, M.; Lloret, F. Dicopper(II) Metallacyclophanes as Multifunctional Magnetic Devices: A Joint Experimental and Computational Study. Acc. Chem. Res. 2015, 48, 510-520.

(6) Tanaka, T.; Osuka, A. Conjugated porphyrin arrays: synthesis, properties and applications for functional materials. Chem. Soc. Rev. 2015, 44, 943-969.

(7) Singh, A. P.; Samuel, P. P.; Roesky, H. W.; Schwarzer, M. C.; Frenking, G.; Sidhu, N. S.; Dittrich, B. A Singlet Biradicaloid Zinc Compound and Its Nonradical Counterpart. J. Am. Chem. Soc. 2013, 135, 7324-7329.

(8) Trojan, K. L.; Hatfield, W. E.; Kepler, K. D.; Kirk, M. L. Strong Exchange Coupling in Lanthanide Bis-(phthalocyaninato) Sandwich Compounds. J. Appl. Phys. 1991, 69, 6007-6009.

(9) Hatfield, W. E.; Singh, P.; Nepveu, F. Structure and Magnetic Properties of $\mathrm{Bis}(\mathrm{N}, \mathrm{N}$-diisopropyldithiocarbamato)copper(II). Inorg. Chem. 1990, 29, 4214-4217.

(10) Kirk, M. L.; Shultz, D. A. Transition Metal Complexes of Donor-Acceptor Biradicals. Coord. Chem. Rev. 2013, 257, 218-233.

(11) Closs, G. L.; Forbes, M. D. E.; Piotrowiak, P. Spin and Reaction Dynamics in Flexible Polymethylene Biradicals as Studied by EPR, NMR, Optical Spectroscopy, and Magnetic Field Effects. Measurements and Mechanisms of Scalar Electron Spin-Spin Coupling. J. Am. Chem. Soc. 1992, 114, 3285-3294.

(12) Ishimaru, Y.; Kitano, M.; Kumada, H.; Koga, N.; Iwamura, H. Regiospecificity in the Exchange Coupling of the Spins of Copper(II) Ion Coordinated with the Ring Nitrogen Atoms andN-tertButylaminoxyl Radical Attached as a Substituent on the Pyridine andN-Phenylimidazole Rings. Inorg. Chem. 1998, 37, 2273-2280.

(13) Lin, V.; DiMagno, S.; Therien, M. Highly conjugated, acetylenyl bridged porphyrins: new models for light-harvesting antenna systems. Science 1994, 264, 1105-1111.

(14) Angiolillo, P. J.; Lin, V. S.-Y.; Vanderkooi, J. M.; Therien, M. J. EPR Spectroscopy and Photophysics of the Lowest Photoactivated Triplet State of a Series of Highly Conjugated (Porphinato)Zn Arrays. J. Am. Chem. Soc. 1995, 117, 12514-12527.

(15) Shediac, R.; Gray, M. H. B.; Uyeda, H. T.; Johnson, R. C.; Hupp, J. T.; Angiolillo, P. J.; Therien, M. J. Singlet and Triplet Excited States of Emissive, Conjugated Bis(porphyrin) Compounds Probed by Optical and EPR Spectroscopic Methods. J. Am. Chem. Soc. 2000, 122, 7017-7033.

(16) Fletcher, J. T.; Therien, M. J. Strongly Coupled Porphyrin Arrays Featuring Both $\pi$-Cofacial and Linear- $\pi$-Conjugative Interactions. Inorg. Chem. 2002, 41, 331-341.

(17) Susumu, K.; Therien, M. J. Decoupling Optical and Potentiometric Band Gaps in $\pi$-Conjugated Materials. J. Am. Chem. Soc. 2002, 124, 8550-8552.

(18) Duncan, T. V.; Susumu, K.; Sinks, L. E.; Therien, M. J. Exceptional Near-Infrared Fluorescence Quantum Yields and ExcitedState Absorptivity of Highly Conjugated Porphyrin Arrays. J. Am. Chem. Soc. 2006, 128, 9000-9001.

(19) Susumu, K.; Frail, P. R.; Angiolillo, P. J.; Therien, M. J. Conjugated Chromophore Arrays with Unusually Large Hole Polaron Delocalization Lengths. J. Am. Chem. Soc. 2006, 128, 8380-8381.

(20) Wang, R.; Ko, C.-H.; Brugh, A. M.; Bai, Y.; Forbes, M. D. E.; Therien, M. J. Topology, Distance, and Orbital Symmetry Effects on Electronic Spin-Spin Couplings in Rigid Molecular Systems: Implications for Long-Distance Spin-Spin Interactions. J. Phys. Chem. A 2020, 124, 7411-7415.

(21) Viere, E. J.; Qi, W.; Stanton, I. N.; Zhang, P.; Therien, M. J. Driving high quantum yield NIR emission through proquinoidal linkage motifs in conjugated supermolecular arrays. Chem. Sci. 2020, $11,8095-8104$.

(22) Castellano, M.; Fortea-Pérez, F. R.; Stiriba, S.-E.; Julve, M.; Lloret, F.; Armentano, D.; De Munno, G.; Ruiz-Garc1, R.; Cano, J. Very Long-Distance Magnetic Coupling in a Dicopper(II) Metallacyclophane with Extended $\pi$-Conjugated Diphenylethyne Bridges. Inorg. Chem. 2011, 50, 11279-11281.
(23) Ferrando-Soria, J.; Castellano, M.; Yuste, C.; Lloret, F.; Julve, M.; Fabelo, O.; Ruiz-Pérez, C.; Stiriba, S.-E.; Ruiz-García, R.; Cano, J. Long-Distance Magnetic Coupling in Dinuclear Copper(II) Complexes with Oligo-Para-Phenylenediamine Bridging Ligands. Inorg. Chim. Acta 2010, 363, 1666-1678.

(24) Wang, R.; Brugh, A. M.; Rawson, J.; Therien, M. J.; Forbes, M. D. E. Alkyne-Bridged Multi[Copper(II) Porphyrin] Structures: Nuances of Orbital Symmetry in Long-Range, Through-Bond Mediated, Isotropic Spin Exchange Interactions. J. Am. Chem. Soc. 2017, 139, 9759-9762.

(25) Forbes, M. D. E.; Jarocha, L. E.; Sim, S.; Tarasov, V. F. TimeResolved Electron Paramagnetic Resonance Spectroscopy: History, Technique, and Application to Supramolecular and Macromolecular Chemistry. Adv. Phys. Org. Chem. 2013, 47, 1-83.

(26) Steiner, U. E.; Ulrich, T. Magnetic Field Effects in Chemical Kinetics and Related Phenomena. Chem. Rev. 1989, 89, 51-147.

(27) Salikhov, K. M.; Molin, Y. N.; Sagdeev, R. Z.; Buchachenko, A. L. Spin Polarization and Magnetic Field Effects in Radical Reactions; Elsevier: Amsterdam, 1984.

(28) Forbes, M. D. E. Time-Resolved EPR Spectroscopy of Flexible Biradicals. R. Soc. Chem. Spec. Period. Reports 2012, 23, 58-78.

(29) Forbes, M. D. E.; Ruberu, S. R. Time-Resolved EPR Spectra of Short Biradicals $(1,3-$ to $1,8-)$ in SolutionThe Interconnecting Roles of the External Magnetic Field, Dipolar Coupling, Exchange Interaction, and Molecular Motion in the Spin Polarization and Relaxation Mechanisms. J. Phys. Chem. 1993, 97, 13223-13233.

(30) Brilliantov, N. V.; Denisov, V. P.; Krapivsky, P. L. Generalized Stokes-Einstein-Debye relation for charged Brownian particles in solution. Phys. A 1991, 175, 293-304.

(31) Kivelson, D. Theory of ESR Linewidths of Free Radicals. J. Chem. Phys. 1960, 33, 1094-1106.

(32) Wilson, R.; Kivelson, D. ESR Linewidths in Solution. IV. Experimental Studies of Anisotropic and Spin-Rotational Effects in Copper Complexes. J. Chem. Phys. 1966, 44, 4445-4452.

(33) Closs, G. L.; Forbes, M. D. E.; Norris, J. R. Spin-Polarized Electron Paramagnetic Resonance Spectra of Radical Pairs in Micelles. Observation of Electron Spin-Spin Interactions. J. Phys. Chem. 1987, 91, 3592-3599.

(34) Closs, G. L.; Forbes, M. D. E. EPR spectroscopy of electron spin-polarized biradicals in liquid solutions: technique, spectral simulation, scope, and limitations. J. Phys. Chem. 1991, 95, 19241933.

(35) Tarasov, V. F.; Jarocha, L. E.; Avdievich, N. I.; Forbes, M. D. E. TREPR Spectra of Micelle-Confined Spin Correlated Radical Pairs: I. Molecular Motion and Simulations. Photochem. Photobiol. Sci. 2014, 13, 439-453.

(36) Tarasov, V. F.; Jarocha, L. E.; Forbes, M. D. E. TREPR Spectra of Micelle-Confined Spin Correlated Radical Pairs: II. Spectral Decomposition and Asymmetric Line Shapes. Photochem. Photobiol. Sci. 2014, 13, 454-463.

(37) Avdievich, N. I.; Forbes, M. D. E. Dynamic Effects in SpinCorrelated Radical Pair Theory: J Modulation and a New Look at the Phenomenon of Alternating Line Widths in the EPR Spectra of Flexible Biradicals. J. Phys. Chem. 1995, 99, 9660-9667.

(38) Forbes, M. D. E.; Avdievich, N. I.; Ball, J. D.; Schulz, G. R. Chain Dynamics Cause the Disappearance of Spin-Correlated Radical Pair Polarization in Flexible Biradicals. J. Phys. Chem. 1996, 100, 13887-13891.

(39) Avdievich, N. I.; Dukes, K. E.; Forbes, M. D. E.; DeSimone, J. M. Time-Resolved EPR Study of a 1,9-Flexible Biradical Dissolved in Liquid Carbon Dioxide. Observation of a New Spin-Relaxation Phenomenon: Alternating Intensities in Spin-Correlated Radical Pair Spectra. J. Phys. Chem. A 1997, 101, 617-621.

(40) Buchachenko, A. L.; Golubev, V. A.; Medzhidov, A. A.; Rosantsev, E. G. Teor. Eksp. Khim. 1965, 1, 249.

(41) Dupeyre, R. M.; Lemaire, H.; Rassat, A. Nitroxides. XIV. A Stable Biradical in the Nitroxide Series. J. Am. Chem. Soc. 1965, 87, 3771 . 
(42) Hudson, A.; Luckhurst, G. R. Electron resonance line shapes of radicals in solution. Chem. Rev. 1969, 69, 191-225.

(43) Toyama, N.; Asano-Someda, M.; Kaizu, Y. EPR Spectra of Gable-Type Copper(II) Porphyrin Dimers in Fluid Solution: Extraction of Exchange Interaction in Weakly Coupled Doublet Pairs. Mol. Phys. 2003, 101, 733-742.

(44) Roychoudhury, S.; Sanvito, S.; O’Regan, D. D. Neutral Excitation Density-Functional Theory: An Efficient and Variational First-Principles Method for Simulating Neutral Excitations in Molecules. Sci. Rep. 2020, 10, 8947.

(45) Lin, V. S.-Y.; Therien, M. J. The Role of Porphyrin-toPorphyrin Linkage Topology in the Extensive Modulation of the Absorptive and Emissive Properties of a Series of Ethynyl- and Butadiynyl-Bridged Bis- and Tris(Porphinato)Zinc Chromophores. Chem.-Eur. J. 1995, 1, 645.

(46) Winters, M. U.; Kärnbratt, J.; Eng, M.; Wilson, C. J.; Anderson, H. L.; Albinsson, B. Photophysics of a Butadiyne-Linked Porphyrin Dimer: Influence of Conformational Flexibility in the Ground and First Singlet Excited State. J. Phys. Chem. C 2007, 111, 7192-7199.

(47) Peeks, M. D.; Neuhaus, P.; Anderson, H. L. Experimental and Computational Evaluation of the Barrier to Torsional Rotation in a Butadiyne-Linked Porphyrin Dimer. Phys. Chem. Chem. Phys. 2016, $18,5264-5274$.

(48) Richert, S.; Kuprov, I.; Peeks, M. D.; Suturina, E. A.; Cremers, J.; Anderson, H. L.; Timmel, C. R. Quantifying the Exchange Coupling in Linear Copper Porphyrin Oligomers. Phys. Chem. Chem. Phys. 2017, 19, 16057-16061.

(49) Rubtsov, I. V.; Susumu, K.; Rubtsov, G. I.; Therien, M. J. Ultrafast Singlet Excited-State Polarization in Electronically Asymmetric Ethyne-Bridged Bis[(porphinato)zinc(II)] Complexes. J. Am. Chem. Soc. 2003, 125, 2687-2696. 